\title{
Planar and cagelike structures of gold clusters: Density-functional pseudopotential calculations
}

\author{
Eva M. Fernándeq \\ Dpto. de Física Teórica, Atómica y Óptica, Universidad de Valladolid, E-47011 Valladolid, Spain. \\ José M. Soler \\ Dpto. de Física de la Materia Condensada, Universidad Autónoma de Madrid, E-28049 Madrid, Spain. \\ Luis C. Balbás \\ Dpto. de Física Teórica, Atómica y Óptica, Universidad de Valladolid, E-47011 Valladolid, Spain.
}

(Dated: September 14, 2018)

\begin{abstract}
We study why gold forms planar and cage-like clusters while copper and silver do not. We use density functional theory and norm-conserving pseudo-potentials with and without a scalar relativistic component. For the exchange-correlation (xc) functional we use both the generalized gradient (GGA) and the local density (LDA) approximations. We find that planar $\mathrm{Au}_{n}$ structures, with up to $n=11$, have lower energy than the three-dimensional isomers only with scalar-relativistic pseudopotentials and the GGA. In all other calculations, with more than 6 or 7 noble metal atoms, we obtain three dimensional structures. However, as a general trend we find that planar structures are more favorable with GGA than with LDA. In the total energy balance, kinetic energy favors planar and cage structures, while xc-energy favors 3D structures. As a second step, we construct cluster structures having only surface atoms with $\mathrm{O}_{h}, \mathrm{~T}_{d}$, and $\mathrm{I}_{h}$ symmetry. Then, assuming one valence electron per atom, we select those with $2(l+1)^{2}$ electrons (with $l$ integer), which correspond to the filling of a spherical electronic shell formed by node-less one electron wave functions. Using scalar relativistic GGA molecular dynamics at $T=600 \mathrm{~K}$, we show that the cage-like structures of neutral $\mathrm{Au}_{32}, \mathrm{Au}_{50}$, and $\mathrm{Au}_{162}$ are meta-stable. Finally, we calculate the static polarizability of the two lowest energy isomers of $\mathrm{Au}_{n}$ clusters as a means to discriminate isomers with planar (or cagelike) geometry from those with compact structures. We also fit our data to a semi-empirical relation for the size dependent polarizability which involves the effective valence and the kinetic energy components for the homogeneous and inhomogeneous electron density. Analyzing that fit, we find that the dipole polarizability of gold clusters with planar and cage-like structures corresponds to the linear response of 1.56 delocalized valence electrons, suggesting a strong screening of the valence interactions due to the $d$-electrons.
\end{abstract}

PACS numbers: $36.40 . \mathrm{Cg}, 36.40 . \mathrm{Qv}$

\section{INTRODUCTION}

Small clusters of metal atoms behave differently than the bulk matter, because each additional atom, or even each additional electron, can drastically change their electronic and geometrical properties 1 . The noble metals clusters, with valence electron filling $n d^{10}(n+1) s^{1}$, differ from the simple $s$-orbital alkali metals ${ }^{2.3}$, but also present striking differences among $\mathrm{Cu}, \mathrm{Ag}$, and $\mathrm{Au}^{4.5}$. Well established structural differences among $\mathrm{Au}_{n}^{\nu}(\nu=0, \pm 1)$ and $\mathrm{Ag}_{n}^{\nu}$ or $\mathrm{Cu}_{n}^{\nu}$ clusters are the following: $\left.i\right) \mathrm{Au}_{n}^{\nu}$ clusters, specially the anions $(\nu=-1)$, adopt planar structures up to larger sizes than $\mathrm{Ag}$ and $\mathrm{Cu}$ clusters, as demonstrated by combined experimental and theoretical studies $\frac{6.7 .8 .9}{} ; \mathrm{ii}$ ) experimental photo-electron spectra for noble metal clusters with 55 atoms 10 indicate that silver and copper adopt some symmetry, preferably icosahedral, whereas the pattern for $\mathrm{Au}_{55}$ corresponds to an amorphous structure $\frac{11}{}$; $i$ ii) anionic and neutral $\mathrm{Au}_{20}$ show a tetrahedral $\mathrm{T}_{d}$ geometry ${ }^{4.12 .13}$, but $\mathrm{Ag}_{20}$ and $\mathrm{Cu}_{20}$ have amorphous-like compact $\mathrm{C}_{s}$ structures; $i v$ ) an icosahedral cage-like structure has been found to be very stable for $\mathrm{Au}_{32}{ }^{14,15}$, but not for silver and copper ${ }^{14,16}$.
Other meta-stable cage-like structures for gold clusters have been proposed recently 16.17 .

The differences between $\mathrm{Au}$ and other noble metal clusters are usually attributed to relativistic effects ${ }^{5}$, which stabilize the $6 s$ orbital and destabilize the $5 d$ one, favoring the hybridization of these orbitals. However, although $\mathrm{Pt}$ shows as strong relativistic effects as $\mathrm{Au}^{2}$, it has been shown that the competition between planar and $3 \mathrm{D}$ structures of $\mathrm{Pt}$ clusters is not affected by relativistic effects $\frac{18}{18}$. Notice that $\mathrm{Pt}_{7}$ is three-dimensional $(3 \mathrm{D})^{19.20}$, like $\mathrm{Ag}_{7}$ or $\mathrm{Cu}_{7}$, but $\mathrm{Au}_{7}$ is planar. Notice also that the largest $s$-orbital contraction due to relativistic effects occurs in $\mathrm{Au}^{21}$. Consideration of the spin-orbit coupling does not alter the relative stability of scalar relativistic structures of $\mathrm{Au}_{n}$ clusters with $n \leq 20$, but it increases the binding energy by about $0.08 \mathrm{eV} /$ atom $(1.85$ $\mathrm{kcal} / \mathrm{mol})^{22}$.

Comparison of density functional theory (DFT) results for $\mathrm{Au}_{6}$ and $\mathrm{Au}_{8}$ at several levels of theory (that is, different exchange-correlation functionals), with results from quantum chemical calculations using second-order perturbation theory (MP2) or coupled cluster methods $(\operatorname{CCSD}(\mathrm{T}))$, indicate that DFT predicts planar struc- 
tures, but MP2 and $\operatorname{CCSD}(\mathrm{T})$ predict the lowest energy $\mathrm{Au}_{8}$ isomer to be non-planar by $26.6 \mathrm{kcal} / \mathrm{mol}$ and 1.5 $\mathrm{kcal} / \mathrm{mol}$, respectively ${ }^{23}$. Another recent calculation ${ }^{24}$, using ab-initio correlated-level theory, predicts $\mathrm{Au}_{8}$ to be planar.

Concerning DFT calculations, we notice that the type of exchange-correlation (xc) functional has a decisive influence on the structural properties of gold clusters, but it is not so critical for silver and copper clusters ${ }^{25}$. Thus, first principles calculations by means of the SIESTA $\operatorname{code}^{26}$ with scalar relativistic pseudopotentials, found planar structures of neutral $\mathrm{Au}_{n}$ clusters with up to 6 atoms using the local density approximation (LDA) for the xc-functiona 27 , and up to 10 atoms using the generalized gradient approximation $(\mathrm{GGA}) \underline{\underline{4}}$. Such a disimilar result was corroborated by a variety of different pseudopotential and all-electron scalar relativistic DFT calculations using $\mathrm{LDA}^{28.29}$ and/or GGA ${ }^{29.30}$.

In a recent paper, Grönbeck and Broqvist ${ }^{31}$ compared the different contributions to the binding energy of several planar and 3D structures of $\mathrm{Au}_{8}$ and $\mathrm{Cu}_{8}$ clusters, optimized within GGA and LDA. They found that planar $\mathrm{Au}_{8}$ isomers have a significative smaller kinetic energy than 3D ones, which was attributed to $d$-electron delocalization. A correlation between strong $s-d$ hybridization and high stability of planar structures was found in ref. 5 for noble metal heptamers, but does not appear to be a general tendency of small Au clusters. Instead, the preference of planar configurations for $\mathrm{Au}_{8}$ isomers was attributed to a sizeable $d$ - $d$ overlap and to $d$-electron delocalization ${ }^{31}$.

Recently, the $\sigma$-aromaticity in saturated inorganic rings was examined ${ }^{32}$. Evidence for $d$-orbital aromaticity in square planar noble metal clusters ${ }^{33}$ and in triangular gold rings ${ }^{34}$ was presented also recently. The spherical $\pi$ aromaticity of $\mathrm{I}_{h}$ symmetrical gold fullerenes fulfilling a generalized $2(l+1)^{2} s$-electron rule $e^{35}$, led Johansson 14 to explain the extra stability of a cage-like $\mathrm{Au}_{32}$ cluster with $\mathrm{I}_{h}$ symmetry compared to other space-filling isomers.

A rough estimation of the photoabsorption response of several isomers of $\mathrm{Au}_{32}$ and $\mathrm{Au}_{42} \frac{36}{}$, suggests that the cage-like structures could be clearly distinguished from space filling isomers in optical absorption experiments. However, it is difficult to separate in these spectra the spectral features due to symmetry from the features due to empty-cage effects. In this paper we will compare the calculated static dipole polarizability of planar and cagelike $\mathrm{Au}_{n}$ clusters with those for 3D and compact isomers.

In section III we outline the first principles method used in our calculations. Results are presented and discussed in section III In subsection IIIA we compare GGA and LDA equilibrium structures of $\mathrm{Au}_{n}$ clusters with $6 \leq n \leq 9$. We will focus on the relation of $2 \mathrm{D}$ or $3 \mathrm{D}$ isomers with the delocalization of $d$ electrons, following the ideas of Grönbeck and Broqvist 31 . In subsection IIIB we contrast again LDA and GGA predictions for the stability of magic cage-like structures $\mathrm{Au}_{18}, \mathrm{Au}_{20}$, $\mathrm{Au}_{32}, \mathrm{Au}_{50}$, and $\mathrm{Au}_{160}$, compared to amorphous-like fill- ing space isomers. The stability of these cage-like structures against molecular dynamics at constant temperature $(600 \mathrm{~K})$, and against the loss or gain of one electron is also tested. In subsection IIIC we investigate the use of the calculated static polarizability as a physical property sensible to the cluster structure. In section [V] we will present our conclusions.

\section{COMPUTATIONAL PROCEDURE}

We use the first-principles code SiESTA 26 to solve fully self-consistently the standard Kohn-Sham equations 37 of DFT within the GGA as parametrized by Perdew, Burke and Ernzerhof ${ }^{38}$, and within the LDA as parametrized by Perdew and Zunger ${ }^{39}$. For each xc-approximation we use a norm conserving scalar relativistic pseudopotential ${ }^{40}$ in its fully nonlocal form ${ }^{41}$, generated from the Au atomic valence configurations $5 d^{10} 6 s^{1} 6 p^{0}$, and core radii which we have tested and reported in previous works 4.42 . Flexible linear combinations of numerical (pseudo) atomic orbitals are used as the basis set, allowing for multiple- $\zeta$ and polarization orbitals. In order to limit the range of the basis pseudoatomic orbitals (PAO), they are slightly excited by a common energy shift $(0.01 \mathrm{eV}$ in this work), and truncated at the resulting radial node ${ }^{43}$. In the present calculations we used a double- $\zeta 5 p, 6 s$-basis, with maximum cutoff radius $7.62 \mathrm{Bohr}$. The basis functions and the electron density are projected onto a uniform real space grid in order to calculate the Hartree and exchangecorrelation potentials and matrix elements. The grid fineness is controlled by the energy cutoff of the plane waves that can be represented in it without aliasing (120 Ry in this work).

To obtain the equilibrium geometries, an unconstrained conjugate-gradient structural relaxation using the DFT forces 44 was performed for several initial cluster structures (typically more than ten), suggested by the several geometries for $\mathrm{Au}_{n}, \mathrm{Au}_{n}^{-}$, and $\mathrm{Au}_{n}^{+}$isomers obtained previously ${ }^{4}$.

The static dipole polarizability of a cluster can be obtained by using the standard numerical finite field perturbation method, in which the field dependent energy is expanded with respect to an external uniform electric field $\mathbf{F}$,

$$
E=E^{0}-\mu_{i} F_{i}-\frac{1}{2} \alpha_{i j} F_{i} F_{j}-\ldots,
$$

where $i, j$ are cartesian coordinates and the dipole moment and the static dipole polarizability are obtained as energy derivatives, $\mu_{i}=-\left.\frac{\partial E}{\partial F_{i}}\right|_{\mathbf{F}=0}$, and $\alpha_{i j}=$ $-\left.\frac{\partial^{2} E}{\partial F_{i} \partial F_{j}}\right|_{\mathbf{F}=0}$, respectively. The external electric field values used in our calculations were (in a.u.) $|\mathbf{F}|=0.000$, $0.001,0.006,0.010,0.014$, and 0.018 . The energies calculated for these values were fitted to a polynomial expansion to obtain the first-order and second-order derivatives of energies with respect to the electric field strength. The mean polarizability is calculated as, $\bar{\alpha}=\operatorname{Tr}\left(\alpha_{i j}\right) / 3$. 


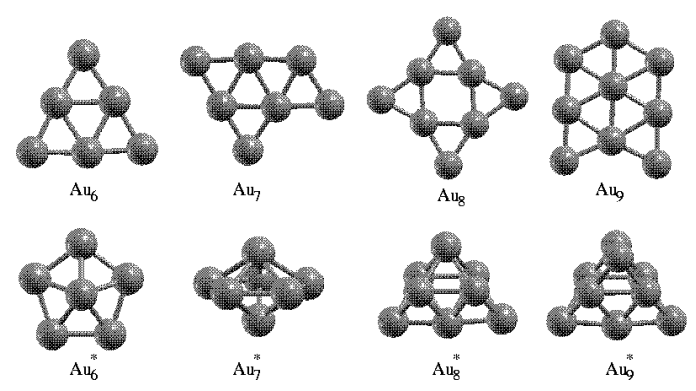

FIG. 1: (Color online) Equilibrium geometry of the lowest energy isomers of gold clusters having planar $\left(\mathrm{Au}_{n}\right.$, upper row) or three dimensional $\left(\mathrm{Au}_{n}^{*}\right.$, lower row) geometry as resulting from LDA and GGA scalar relativistic calculations. For GGA the ground state is the planar $\mathrm{Au}_{n}$ isomer. For LDA the ground state is the $3 \mathrm{D} \mathrm{Au}_{n}^{*}$ isomer, except for $n=$ 6, whose structure is also the planar $\mathrm{Au}_{6}$.

\section{RESULTS AND DISCUSSIONS}

\section{A. Planarity and $d$-electron delocalization in $\mathbf{A u} \mathbf{u}_{n}$}

The onset of three-dimensional structures of neutral $\mathrm{Au}_{n}$ clusters was calculated at $n=11$ within $\mathrm{GGA}^{4}$, and at $n=7$ within $\mathrm{LDA}^{27}$. In this work we compare the planar and 3D lowest energy isomers of $\mathrm{Au}_{n}(6 \leq n \leq 9)$ calculated within the SiESTA code ${ }^{26}$, and using $\operatorname{LDA}^{39}$ and $\mathrm{GGA}^{38}$ for xc-functionals (with the corresponding LDA and GGA scalar relativistic pseudo-potentials). Figure 1 shows our results for the geometry of these isomers. The lower energy isomer is the planar one for GGA, and the $3 \mathrm{D}$ one for LDA, except for $n=6$. Table \ gives the binding energy difference between $2 \mathrm{D}$ and $3 \mathrm{D}$ isomers, $\Delta E_{b}=E_{b}\left(\mathrm{Au}_{n}\right)-E_{b}\left(\mathrm{Au}_{n}^{*}\right)$, for the relativistic and nonrelativistic calculations. We see that GGA leads to planar structures, but LDA favors 3D structures for $n \geq 7$ clusters. Thus, although the planarity of $\mathrm{Au}_{7}$ compared to the $3 \mathrm{D}$ structures of $\mathrm{Ag}_{7}$ or $\mathrm{Cu}_{7}$ was attributed to relativistic effects ${ }^{5}$, the observed planarity of Au clusters ${ }^{6.7 .8}$ is accounted for only using the GGA theory. We have also optimized the $\mathrm{Au}_{n}$ structures in Figure 1 within GGA and LDA non relativistic pseudopotentials, resulting that the 3D structures become more stable energetically than the planar ones, except for $n=6$ within GGA, as shown in the last row of Table \

TABLE I: The binding energy difference $\Delta E_{b}=E_{b}\left(\mathrm{Au}_{n}\right)-$ $E_{b}\left(\mathrm{Au}_{n}^{*}\right)$, in $\mathrm{eV}$, between the $2 \mathrm{D}$ and $3 \mathrm{D}$ isomers of the gold clusters represented in Fig. 1] optimized within LDA and GGA xc-functionals using relativistic and non-relativistic (NR) pseudopotentials.

\begin{tabular}{lccccccccc}
\hline \hline & \multicolumn{2}{c}{$\mathrm{Au}_{6}$} & \multicolumn{2}{c}{$\mathrm{Au}_{7}$} & \multicolumn{2}{c}{$\mathrm{Au}_{8}$} & \multicolumn{2}{c}{$\mathrm{A} u_{9}$} \\
& LDA & GGA & LDA & GGA & LDA & GGA & LDA & GGA \\
\hline$\Delta \mathrm{E}_{b}$ & 0.14 & 0.15 & -0.02 & 0.03 & -0.06 & 0.01 & -0.04 & 0.01 \\
$\Delta \mathrm{E}_{b}^{N R}$ & -0.04 & 0.02 & -1.42 & -1.11 & -1.64 & -1.37 & -1.29 & -0.98 \\
\hline \hline
\end{tabular}

Table gives the various energy differences (total, kinetic, Coulomb, and exchange-correlation) between the second and first energy isomers of scalar relativistic $\mathrm{Au}$ and $\mathrm{Cu}$ clusters with $6 \leq n \leq 9$ atoms. We will denote compact 3D structures with an asterisk, while its absence indicates planar and cage-like geometries. The geometry of the $\mathrm{Au}$ isomers is given in Figure 1 The geometries of $\mathrm{Cu}_{6}$ and $\mathrm{Cu}_{6}^{*}$ are similar to those of $\mathrm{Au}_{6}$ and $\mathrm{Au}_{6}^{*}$ respectively. For $\mathrm{Cu}_{n}$ with $n=7,8,9$, the geometries of the two lowest energy isomers are both $3 \mathrm{D}$, and are taken from our previous work ${ }^{4}$. We can see in Table that planar structures have smaller kinetic energy than 3D isomers, and larger for LDA than for GGA. Adding kinetic and Coulomb energies, the 2D structures became more stable energetically than the 3D ones. On the other hand, the xc-energy is more negative (it contributes more to the binding energy) for 3D than for planar structures. Both effects are stronger within the LDA but, in the balance of total energy difference, the loss of kinetic energy in planar structures dominates over the increase of xcenergy when using GGA, but the opposite occurs with LDA. Thus, as a whole, GGA (LDA) favors 2D (3D) structures of gold clusters. On the other hand, for $\mathrm{Cu}_{6}$ and $\mathrm{Cu}_{6}^{*}$ isomers, which have the same geometry as $\mathrm{Au}_{6}$ and $\mathrm{Au}_{6}^{*}$, the change in kinetic and Coulomb energies is not so noticeable as in gold.

For $\mathrm{Cu}_{n}$ with $n=7-9$, whose first and second isomer geometries are all 3D, the change in exchange-correlation energy, $\Delta_{x c}$, can be positive or negative. For $\mathrm{Cu}_{8}$, whose electronic properties can be described approximately by the spherical jellium mode ${ }^{4}$, we see that the sum of changes in kinetic and Coulomb energies roughly cancel, resulting that the change in the total energy is ruled by the change in xc-energy, as in the jellium mode 15 .

The loss of kinetic energy in planar gold clusters with respect to their $3 \mathrm{D}$ isomers was attributed to electron delocalization ${ }^{31}$, but is not easy to reconcile that delocalization with the simultaneous confinement in two dimensions. On the other hand, the xc energy becomes less negative for planar configurations, which is also not clearly related to delocalization and confinement in $2 \mathrm{D}$ gold clusters. We observe that the calculated average bond length, $d_{a v}$, is larger for 3D than for 2D isomers. Specifically, the difference between $d_{a v}$ of 3D and 2D $\mathrm{Au}_{n}$ isomers with $n=6,7,8,9$, is (in $\AA$ ) $0.04,0.11,0.13,0.09$ $(0.05,0.11,0.09,0.09)$ for the LDA (GGA) calculation.

The kinetic energy of the electron gas in two dimensions is about one half of the $3 \mathrm{D}$ case at the same density parameter, $r_{s}$, while the exchange energy in $2 \mathrm{D}$ is slightly larger than in $3 \mathrm{D}^{46.47}$. However, the correlation energy, at least in the RPA, is much larger in 2D than in $3 \mathrm{D}^{46}$. This consideration points again to the importance of good exchange-correlation functionals in dealing with the DFT structural description of gold clusters. 
TABLE II: Total, kinetic, Coulomb, and exchange- correlation energy differences $\left(\Delta E_{i}=E_{i}\left(\mathrm{Au}_{n}^{*}\right)-E_{i}\left(\mathrm{Au}_{n}\right)\right)$, in $\mathrm{eV}$, between the second isomer $\left(3 \mathrm{D}, \mathrm{Au}_{n}^{*}\right)$ and first isomer $(2 \mathrm{D}$, $\mathrm{Au}_{n}$ ) of gold clusters with the structures of Fig 1 For $\mathrm{Cu}_{n}$ the first and second isomers are $3 \mathrm{D}$, except for $\mathrm{Cu}_{6}$, where the two isomers are similar to $\mathrm{Au}_{6}$ and $\mathrm{Au}_{6}^{*}$. The $\mathrm{Cu}_{n}$ geometries are taken from ref. 4 .

\begin{tabular}{lrrrrrrrrr}
\hline \hline & \multicolumn{2}{c}{$\Delta E_{\text {tot }}$} & \multicolumn{2}{c}{$\Delta E_{\text {kin }}$} & \multicolumn{2}{c}{$\Delta E_{\text {Coul }}$} & \multicolumn{2}{c}{$\Delta E_{x c}$} \\
& LDA & GGA & LDA & GGA & LDA & GGA & LDA & GGA \\
\hline $\mathrm{Au}_{6}$ & 0.86 & 0.88 & 11.01 & 10.63 & -9.62 & -9.42 & -0.53 & -0.33 \\
$\mathrm{Au}_{7}$ & -0.15 & 0.24 & 12.49 & 10.34 & -11.12 & -9.52 & -1.53 & -0.57 \\
$\mathrm{Au}_{8}$ & -0.46 & 0.04 & 7.37 & 7.03 & -6.53 & -6.80 & -1.30 & -0.19 \\
$\mathrm{Au}_{9}$ & -0.34 & 0.07 & 5.07 & 4.63 & -4.50 & -4.52 & -0.91 & -0.04 \\
\hline $\mathrm{Cu}_{6}$ & 0.03 & & 1.03 & & -078 & & -0.22 \\
$\mathrm{Cu}_{7}$ & 0.34 & & 0.20 & & -0.98 & & 1.13 \\
$\mathrm{Cu}_{8}$ & & 0.89 & & 2.10 & & -1.98 & & 0.77 \\
$\mathrm{Cu}_{9}$ & 0.25 & & -1.60 & & 1.87 & & -0.02 \\
\hline \hline
\end{tabular}

\section{B. Magic cage-like structures of gold clusters}

Stable cage-like structures of gold clusters have been predicted recently for $\mathrm{Au}_{32}{ }^{14,15}, \mathrm{Au}_{26}{ }^{48}, \mathrm{Au}_{42}{ }^{49}$, and others ${ }^{16.17}$. On the other hand, although all atoms of $\mathrm{Au}_{20}$ are at the surface, this cluster can be considered as a small piece of bulk fcc gold ${ }^{4,12,13}$.

In this work we construct cage-like atomic structures starting with the Platonic solids with triangular faces - tetrahedron $\left(\mathrm{T}_{d}\right)$, octahedron $\left(\mathrm{O}_{h}\right)$, and icosahedron $\left(\mathrm{I}_{h}\right)$ - which are those allowing compact planar packing. By adding atoms at the intersections of fcc planes in the triangles, we obtain the following sequences for the number of atoms: $n\left(\mathrm{~T}_{d}\right)=4+2 m(m+2), n\left(\mathrm{O}_{h}\right)=6$ $+4 m(m+2)$, and $n\left(\mathrm{I}_{h}\right)=12+10 m(m+2)$, where $m=$ $0,1,2, \ldots$ is the number of atoms inserted in each edge. When we add a central atom to each new triangle, we obtain cage-like structures with $n\left(\mathrm{~T}_{d}\right)=2+6(m+1)^{2}$, $n\left(\mathrm{O}_{h}\right)=2+12(m+1)^{2}$, and $n\left(\mathrm{I}_{h}\right)=2+30(n+1)^{2}$. On the other hand, from the electronic point of view, a cluster with nearly free valence electrons is magic when it has filled electronic shells, having well defined angular momentum, as in the jellium model. As we look for an empty-cage with an approximately spherical surface, orbitals with radial nodes have to be excluded and the only allowed electronic shells are $1 s, 1 p, 1 d, 1 f, \ldots$ This leads to a magic number of electrons $n_{e}=2(l+1)^{2}$, where $l$ is an integer number ${ }^{50}$. Assuming that each noble metal atom contributes with one valence electron, the equality $n=2(l+1)^{2}$ leads to the following magic neutral cage-like $\mathrm{Au}_{n}$ clusters (containing less than 1000 atoms): $n=8\left(\mathrm{~T}_{d}\right), 18\left(\mathrm{O}_{h}\right), 32\left(\mathrm{I}_{h}\right), 50\left(\mathrm{O}_{h}\right), 98\left(\mathrm{~T}_{d}\right)$, $162\left(\mathrm{I}_{h}\right)$, and $578\left(\mathrm{O}_{h}\right)$. Double anionic clusters should obey $n+2=2(l+1)^{2}$ and they appear at $n=6$ and $198\left(\mathrm{O}_{h}\right)$ atoms. Double cationic magic clusters are the solutions of $n-2=2(l+1)^{2}$. However, we exclude the $\mathrm{T}_{d}$ clusters because they are far from spherical. Also

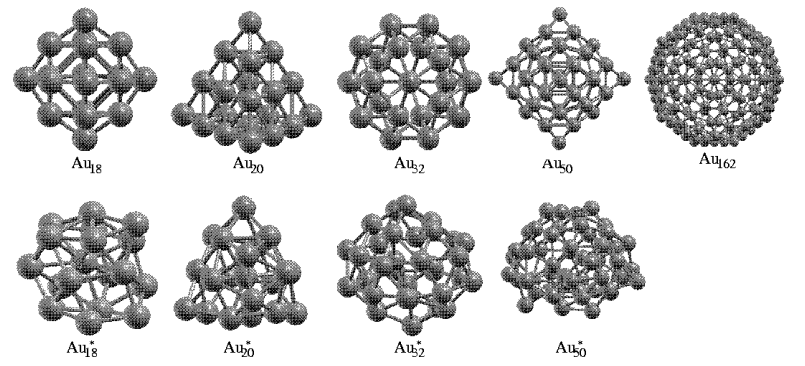

FIG. 2: (Color online) Cage-like $\left(\mathrm{Au}_{n}\right)$ and space filling $\left(\mathrm{Au}_{n}^{*}\right)$ equilibrium isomeric structures of neutral gold clusters with $n$ $=18,20,32,50$, and 162 , except $\mathrm{Au}_{162}^{*}$ which is not optimized in this paper.

$\mathrm{Au}_{20}$ can not be properly considered cage-like because it contains many internal bonds, as similarly occurs to smaller clusters, like $\mathrm{Au}_{18}$. Nevertheless, in the following we will test the estructural and electronic properties of $\mathrm{Au}_{n}$ clusters with 18, 20, 32, 50, and 162 atoms.

TABLE III: Binding energy difference between the cage-like and compact structures of Fig $2 \Delta E_{b}=E_{b}\left(\mathrm{Au}_{n}\right)-E_{b}\left(\mathrm{Au}_{n}^{*}\right)$, optimized with the LDA and GGA approximations.

\begin{tabular}{ccccccccc}
\hline \hline & \multicolumn{2}{c}{$\mathrm{Au}_{18}$} & \multicolumn{2}{c}{$\mathrm{Au}_{20}$} & \multicolumn{2}{c}{$\mathrm{Au}_{32}$} & \multicolumn{2}{c}{$\mathrm{A} u_{50}$} \\
& LDA & GGA & LDA & GGA & LDA & GGA & LDA & GGA \\
\hline$\Delta \mathrm{E}_{b}$ & -0.07 & 0.01 & 0.01 & 0.03 & -0.06 & 0.01 & -0.14 & 0.01 \\
\hline \hline
\end{tabular}

We performed full relaxations of the initial cage-like magic structures and several compact geometries obtained by forcing initially some surface atoms inside these clusters. Fig 2 shows the equilibrium geometries with cage-like and compact structures, obtained after a non exhaustive search, and optimized with forces $\leq 0.01$ $\mathrm{eV} / \AA$ ) at the GGA and LDA levels. The cage-like equilibrium structures were proven to be meta-stable after performing an ab-initio molecular dynamics run at temperature of $600 \mathrm{~K}$ during 1000 steps, each of 2 fs. The binding energy difference between cage-like and compact structures is tabulated in Table【II We see that cage-like GGA structures are slightly more bound than the compact ones. Instead, LDA leads to compact structures without symmetry, except for $\mathrm{Au}_{20}$. The true ground state is not known, however, and improved functionals could lead to compact, ordered or disordered, structures.

As a further test, we calculate the relative stability of cage-like and compact isomers after loss or gain of one electron. Fig[3] shows the total energy difference per atom between cage-like and compact equilibrium structures of cationic, neutral, and anionic clusters with $n=18,20,32$ atoms. We see that only the cation $\mathrm{Au}_{32}^{+}$and the anion $\mathrm{Au}_{20}^{-}$are still cage-like. Interestingly, the lowest energy isomer of both ionic clusters, $\mathrm{Au}_{18}^{+}$and $\mathrm{Au}_{18}^{-}$, is not cagelike, contrary to the neutral $\mathrm{Au}_{18}$.

In Fig 4 we compare the density of states (DOS) of the cage-like and amorphous structures of neutral $\mathrm{Au}_{n}$ 
$(n=18,20)$. We see that the cage-like geometries show a more structured DOS, with well defined peaks. This is probably due to a higher geometrical symmetry $\left(\mathrm{O}_{h}\right.$ for $\mathrm{Au}_{18}$ and $\mathrm{T}_{d}$ for $\mathrm{Au}_{20}$ ), as shown in a previous work for $\mathrm{Au}_{55}{ }^{10}$. This fact is independent of the GGA or LDA level of theory. The LDA DOS profile tends to be shifted (to lower energies for $\mathrm{Au}_{20}$ ) with respect to the GGA one, and the HOMO-LUMO gap is smaller for LDA than for GGA. On the other hand the HOMO-LUMO gap is considerably larger for cage-like $\mathrm{Au}_{20}$ than for cage-like $\mathrm{Au}_{18}$, indicating that the former is much more stable than the later.

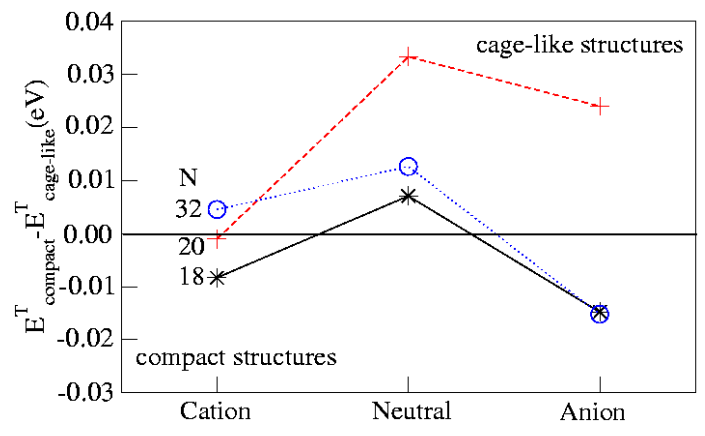

FIG. 3: (Color online) Total GGA energy difference per atom (in $\mathrm{eV}$ ) between the lower energy isomer of compact and cagelike structures for cationic, neutral, and anionic clusters of gold with $n=18$ (stars), 20 (crosses), and 32 (circles) atoms.

\section{Static dipole polarizability}

The minimum polarizability principle states that any system evolves naturally towards a state of minimum polarizability, but exceptions have been reported ${ }^{51}$. In Table $\amalg$ Vare given the results of our scalar-relativistic GGA calculations for the mean polarizability per atom of the two lowest energy states of the Au clusters reported in the subsections above.

The static dipole polarizability of atomic $\mathrm{Au}$ comes to 20.53 a.u. $\left(\right.$ Bohr $\left.^{3}\right)$ for the scalar-relativistic calculation, and 33.06 a.u. for the non-relativistic calculation, which is a clear manifestation of the relativistic size contraction of gold ${ }^{2}$. Our Au polarizability is smaller than other calculated and experimental values quoted in the literature 2.52.53.54. Different estimates of the experimental value are $30 \pm 4$ a.u. $\frac{2}{2}$ and $39.1 \pm 9.8$ a.u $\stackrel{52}{\underline{2}}$. A quantum chemical $\operatorname{CCSD}(\mathrm{T})$ calculation gives 36.06 a.u. $\stackrel{53}{ }$, and a recent CASSCF/CASPT2 relativistic calculation gives 27.9 a.u. ${ }^{52}$.

For noble metal dimers, recent relativistic and nonrelativistic calculations ${ }^{55}$ found that the reduction of the polarizability, due to the relativistic contraction effect, amounts to $39.8 \%$ for $\mathrm{Au}_{2}, 15.8 \%$ for $\mathrm{Ag}_{2}$, and $6 \%$ for $\mathrm{Cu}_{2}$. From the TDDFT study of Castro and coworkers $\$$ the static polarizability of $\mathrm{Au}_{n}$ clusters up to $n=4$ are

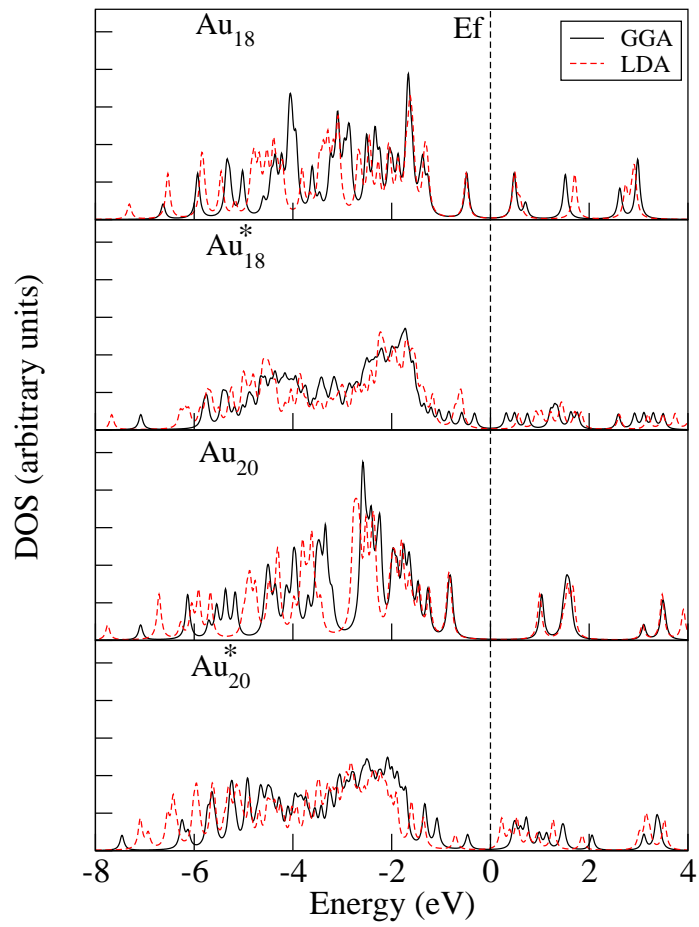

FIG. 4: (Color online) Density of states (DOS) of the lower energy cage-like and amorphous isomers of $\mathrm{Au}$ clusters with 18 and 20 atoms, calculated at the LDA and GGA levels of theory.

affected up to $\sim 2 \%$ by the inclusion of the spin-orbit term.

From the TDDFT calculations of Castro ${ }^{56}$, the static polarizability of the $3 \mathrm{D}$ tetrahedral isomer of $\mathrm{Au}_{4}$ is $2.5 \%$ higher than the one for the planar $\mathrm{D}_{2 h}$ ground state. We obtain a larger difference $(15.5 \%)$ between our two planar $\mathrm{Au}_{4}$ isomers (a rhombus and a triangle with another $\mathrm{Au}$ on top, see ref $\mathrm{f}^{4}$. Our result for the $\mathrm{Au}_{n}$ polarizability with $n=2-9$, is similar to the one calculated by Zhao and coworkers 57 using a finite field perturbation method like ours. However, the polarizability per atom obtained by these authors for planar $\mathrm{Au}_{18}$ and $\mathrm{Au}_{20}$ is much higher than ours for cage-like and compact isomers. On the other hand, the difference between GGA and LDA polarizabilities of planar $\mathrm{Au}_{n}$ clusters $(n=2-20)$ calculated in ref. 57 is less than $2 \%$.

We see in Table IV that the average polarizability per atom of $\mathrm{Au}_{n}$ clusters is remarkably constant, with small odd-even effects for $n \leq 7$. Up to $n=6$ the first and second isomers are planar, and the larger polarizability of the second isomer is due to the larger average $\mathrm{Au}$ $\mathrm{Au}$ bond length. The polarizability of the $\mathrm{Au}_{7}^{*} 3 \mathrm{D}$ isomer is smaller than that of the planar isomer, due to the compact pentagonal bipyramid geometry, and it constitutes one of the exceptions to the minimum polarizability principle ${ }^{51}$. The cage-like structures seem to be another exception to that rule.

The average mean polarizability per atom for the first 
TABLE IV: The third and fourth columns give, respectively, the mean polarizability per atom $\bar{\alpha} / n$ of the first and second isomers of $\mathrm{Au}_{n}$ clusters. The last column gives their difference. For comparison, the first column gives the $\bar{\alpha} / \mathrm{n}$ value calculated by Zhao and coworkers for planar estructures 57 . All polarizabilities are in atomic units $\left(\right.$ bohr $\left.^{3}\right)$.

\begin{tabular}{rcccr}
\hline \hline & & $\bar{\alpha} / n$ & $\bar{\alpha} / n$ & \\
$n$ & Ref. 57 & 1st isomer & 2nd isomer & $\Delta \bar{\alpha}(\%)$ \\
\hline 2 & 29.4 & 28.3 & 29.0 & 2.5 \\
3 & 31.1 & 36.4 & 43.0 & 18.2 \\
4 & 32.7 & 31.5 & 37.2 & 17.9 \\
5 & 34.2 & 32.9 & 46.6 & 41.6 \\
6 & 34.2 & 32.7 & 40.0 & 22.5 \\
7 & 34.3 & 33.9 & 30.9 & -9.0 \\
8 & 36.2 & 34.7 & 36.1 & 4.2 \\
9 & 38.5 & 37.8 & 48.1 & 27.3 \\
\hline 18 & 42.7 & 32.1 & 31.4 & -2.4 \\
20 & 43.7 & 35.2 & 32.9 & -6.4 \\
32 & & 33.9 & 33.5 & -1.3 \\
\hline \hline
\end{tabular}

isomer of $\mathrm{Au}_{n}$ clusters in Table IV is $\bar{\alpha}_{a v} / n=33.57$ a.u.. The jellium model for atomic clusters of monovalent metals fulfills the relation $\alpha_{j e l}=r_{s}^{3} n$, where $r_{s}$ is the radius per electron (in a.u.) of the bulk metal. For Au, with $r_{s}$ $=3.01$ and the GGA, we obtain the ratio $\tilde{\alpha} \equiv \bar{\alpha}_{a v} / \alpha_{j e l}$ $=1.23$. We can calculate a similar ratio for $\mathrm{Li}, \mathrm{Na}, \mathrm{Cu}$, and $\mathrm{Ag}$ clusters using results from experiments and other calculations. Using the experimental values ${ }^{58.59}$ for the dipole polarizability of $\mathrm{Li}\left(r_{s}=3.25\right), \mathrm{Na}\left(r_{s}=3.93\right)$, and $\mathrm{Cu}\left(r_{s}=2.67\right)$ clusters with $n \leq 10$, we obtain the ratios $\tilde{\alpha}_{e x p}(\mathrm{Li})=2.26, \tilde{\alpha}_{\exp }(\mathrm{Na})=2.11$, and $\tilde{\alpha}_{e x p}(\mathrm{Li})=2.0$ for $\mathrm{Li}, \mathrm{Na}$, and $\mathrm{Cu}$, respectively. From the GGA polarizability values calculated in ref. 60 for $\mathrm{Li}$ and $\mathrm{Na}$ clusters with $n \leq 10$, we find $\tilde{\alpha}_{G G A}(\mathrm{Li})=2.47$ and $\tilde{\alpha}_{G G A}(\mathrm{Na})=$ 1.81. For $\mathrm{Cu}$ clusters in the same range of sizes, using the different GGA and LDA polarizability values reported by Yang and Jackson ${ }^{61}$, one has $\tilde{\alpha}_{G G A}(\mathrm{Cu})=1.93-1.98$, and $\tilde{\alpha}_{L D A}(\mathrm{Cu})=1.79$. Using recent calculations ${ }^{17}$ for the polarizability of Ag clusters with $2 \leq n \leq 8$, results in $\tilde{\alpha}_{G G A}(\mathrm{Ag})=1.69$. As a whole, we see that the ratio $\bar{\alpha}_{a v} / \alpha_{\text {jel }}$ is considerably smaller for $\mathrm{Au}$ than for $\mathrm{Li}, \mathrm{Na}$, $\mathrm{Cu}$, and $\mathrm{Ag}$ clusters.

The enhancement of dipole polarizabilty over the classical jellium model (Mie value $\alpha_{j e l}$ ) is directly proportional to the fraction of electronic charge that extends beyond the positive background in the field free system (spill-out) $\frac{1}{*}$. The smaller spill-out for gold with respect to silver and copper can be attributed tentatively to the relativistic contraction of the electronic cloud ${ }^{2.21}$.

We can explore a little more our calculated polarizability values using an extended Thomas-Fermi-Weizsäcker $(\mathrm{TF} \lambda \mathrm{W})$ jellium mode ${ }^{62}$, which predicts the mean polarizability of a cluster with $n_{e}=v n$ valence electrons $(v$
= valence, $n=$ number of atoms) as

$$
\bar{\alpha}=\alpha_{j e l}\left[1+3 \frac{d\left(r_{s}\right)}{r_{s}} n_{e}^{-1 / 3}\left(1+\frac{\alpha_{1}\left(r_{s}\right)}{\alpha_{0}\left(r_{s}\right)} n_{e}^{-1 / 3}\right)\right]
$$

In this expression, $d\left(r_{s}\right)$ is the image plane position (the centroid of the induced electron density for the flat metal surface), and $\alpha_{1}$ and $\alpha_{0}$ are two coefficients dependent of the parameter $\lambda$, which take into account the weight of inhomogeneity-density correction (Weizsäcker term) to the Thomas-Fermi kinetic energy. In the interval $3 \leq r_{s}$ $\leq 4$, it results $\alpha_{1}\left(r_{s}\right) \simeq-0.1$ a.u. independently of the $\lambda$ value (see Fig. 1 of ref. 62), but $\alpha_{0}\left(r_{s}\right)$ is strongly dependent of $\lambda$. In terms of a reduced polarizability, defined by $\alpha_{\text {red }}=\left(\bar{\alpha} / \alpha_{j e l}-1\right) n^{2 / 3}$, we can write eq. 2 as

$$
\alpha_{\text {red }}=A n^{1 / 3}+A B
$$

where $A=\frac{3}{v^{1 / 3}} \frac{d\left(r_{s}\right)}{r_{s}}$, and $B=\frac{1}{v^{2 / 3}} \frac{\alpha_{1}\left(r_{s}\right)}{\alpha_{0}\left(r_{s}\right)}$. By fitting our data in Table IV to eqn. 3 we obtain $\alpha_{\text {red }}=$ $1.15 n^{1 / 3}-1.26$, and using $d(\mathrm{Au})=1.34 \mathrm{a}$.u. for the image plane distance of gold ${ }^{63}$, there results an effective valence $v=1.56$, and a ratio $\frac{\alpha_{1}\left(r_{s}\right)}{\alpha_{0}\left(r_{s}\right)}=-1.46$. For $\alpha_{1}(A u) \simeq$ -0.1 we obtain $\alpha_{0}(A u)=0.07$. Extrapolating the results in Fig. 1 of ref. 62, such a small $\alpha_{0}(A u)$ value corresponds to an extremely small value of the parameter $\lambda$, which means that the contribution of inhomogeneity corrections to the kinetic energy is very small. This result agrees with our conclusion in subsection IIIA about the delocalization of valence electrons in planar gold clusters. On the other hand, the effective valence $v=1.56$ reflects in some way the screening of the dipole response due to the $d$-electrons 56 .

We test also an empirical linear relation between the cubic root of the mean polarizability per atom, $\bar{\alpha}^{1 / 3} / \mathrm{n}$, and the inverse of the ionization potential per atom, $\mathrm{I}_{p}^{-1} / \mathrm{n}$. That relation was tested in ref. 60 for $\mathrm{Li}$ and Na clusters with 2-10 atoms, resulting for both cases (using calculated GGA values, in a.u.) in a proportionality constant close to unity, with a linear correlation coefficient 0.995 . For the lower energy isomers of $\mathrm{Au}$ clusters reported in Table IV there results a proportionality constant 0.925 and a correlation coefficient of 0.984 .

As probed by Yang and Jackson ${ }^{61}$, temperature effects are a possible source of discrepancy between calculated and measured polarizabilities, because calculations are carried out for $0 \mathrm{~K}$ while experiments are conducted at finite temperatures. The existence of a permanent electric dipole in a cluster adds the following temperaturedependent term to the effective polarizability 64 :

$$
\alpha_{e f f}=\bar{\alpha}+\frac{\mu^{2}}{3 k T},
$$

where $\mu$ is the dipole moment and $k$ is the Boltzman constant. The dipole contribution is important at low temperatures for clusters with permanent dipole moments. Using the dipole moments calculated for our GGA lower 
energy isomers, the correction of eqn. 4 to the mean polarizability per atom is (in a.u) 1.39, 0.04, 0.74, 2.33, and 0.09 , for clusters with $3,4,5,6$, and 20 atoms, respectively. This small correction still allows one to discriminate the polarizability of planar and cage-like gold clusters from their isomers.

\section{CONCLUSIONS}

We obtain that, using non-relativistic pseudopotentials, both GGA and LDA predict the onset of three dimensional cluster structures already at $n=6$ for $\mathrm{Cu}_{n}$ and $\mathrm{Ag}_{n}$, and at $n=7$ (6) for $\mathrm{Au}_{n}$. This result changes by considering scalar-relativistic pseudopotentials within GGA, resulting in planar $\mathrm{Au}_{n}$ structures up to $n=11$.

From our scalar-relativistic results for the two lowest energy isomers of $\mathrm{Au}_{n}$ and $\mathrm{Cu}_{n}$ with $n=6-9$ atoms, we find that planar structures have smaller kinetic energy than 3D isomers, and this effect is much larger for gold than for copper clusters. Adding kinetic and Coulomb energies, the $2 \mathrm{D}$ structures became more stable than the $3 \mathrm{D}$ ones, and this effect is more noticeable for LDA than for GGA. On the other hand, the xc-energy is more negative (contribute more to the binding energy) for 3D than for planar structures, and this effect is notably enhanced within LDA. Thus, in the total energy balance, kinetic energy loss favors planar GGA structures, but xc-energy favors LDA 3D structures.

As a second step, we constructed clusters having only surface atoms, and with $\mathrm{O}_{h}, \mathrm{~T}_{d}$, and $\mathrm{I}_{h}$ symmetry. From those, assuming one valence electron per atom, we select the ones having $2(l+1)^{2}$ electrons, which correspond to the filling of a spherical electronic shell formed by nodeless one electron wave functions. We obtain, by means of scalar-relativistic GGA calculations, that these cage- like structures for neutral $\mathrm{Au}_{18}, \mathrm{Au}_{20}, \mathrm{Au}_{32}, \mathrm{Au}_{50}$, and $\mathrm{Au}_{162}$ are meta-stable after moderate $(600 \mathrm{~K})$ constant temperature molecular dynamics. However, after addition or substraction of an electron, only the anion $\mathrm{Au}_{20}^{-}$ and the cation $\mathrm{Au}_{32}^{+}$remain cage-like.

Finally, we calculate the static polarizability of the two lowest energy isomers of $\mathrm{Au}_{n}$ clusters, which are planar $(n=2-9)$ and cage-like $(n=18,20,32)$ for the first isomer and planar $(n=2-6)$ or space filling $3 \mathrm{D}(n=7$ $9,18,20,32)$ for the second isomer. In the range $n=$ $2-9$, the polarizability per atom is smaller for the first isomer than for the second, with the exception of $n=7$, confirming the empirical rule of minimum polarizability. The contrary occurs for cage-like structures, with larger $\bar{\alpha}$ than their space-filing isomers.

We fitted the polarizability of the first isomer of these gold clusters to a semi-empirical relation between the cluster dipole polarizability and its size, which involves the effective atomic valence and the kinetic energy due to homogeneous and to inhomogeneous density components. From that fit we extract a very small value for the kinetic energy component due to inhomogeneous density, which suggest a delocalized character of the valence electrons involved in the dipole response. We also obtain an effective valence charge of 1.56 electrons, reflecting a dipole response with strong screening of the $d$-electrons, as already reported previously ${ }^{56}$.

\section{Acknowledgments}

We want to acknowledge the financial support from grants MAT2005-03415 and BFM2003-03372 of the Spanish Ministery of Science, and from the FEDER of the European Community.
* eva@lcb.fam.cie.uva.es

1 W. Ekardt, Metal Clusters, Wiley, Chichester, 1999.

${ }^{2}$ P. Pyykkö, Angew. Chem. Int. Ed. 43, 4412 (2004).

3 P. Pyykkö, Inorganica Chimica Acta 358, 4113 (2005).

4 E. M. Fernández, J. M. Soler, I. L. Garzón, and L. C. Balbás, Phys. Rev. B 70, 165403 (2004).

${ }^{5}$ H. Häkkinen, M. Moseler, and U. Landman, Phys. Rev. Lett. 89, 033401 (2002).

${ }^{6}$ F. Furche, R. Ahlrich, P. Weis, C. Jacob, S. Gilb, T. Bienweiler, and M. Kappes, J. Chem. Phys. 117, 6982 (2002).

7 P. W. S. Gilb, F. Furche, R. Ahlrichs, and M. M. Kappes, J. Chem. Phys. 116, 4094 (2002).

${ }^{8}$ P. Weis, T. Bierweiler, S. Gilb, and M. M. Kappes, Chem. Phys. Lett. 355, 355 (2002).

9 H. Häkkinen, B. Yoon, U. Landman, X. Li, H.-J. Zhai, and L.-S. Wang, J. Phys. Chem. A 107, 6168 (2003).

10 H. Häkkinen, M. Moseler, O. Kostko, N. Morgner, M. A. Hoffmann, and B. von Issendorff, Phys. Rev. Lett. 93, 093401 (2004).

11 I. L. Garzón, K. Michaelian, M. R. Beltrán, A. Posada-
Amarillas, P. Ordejón, E. Artacho, D. Sáchez-Portal, and J. M. Soler, Phys. Rev. Lett. 81, 1600 (1998).

12 J. Li, X. Li, H.-J. Zhai, and L.-S. Wang, Science 299, 864 (2003).

13 J. Wang, G. Wang, and J. Zhao, Chem. Phys. Lett. 380, 716 (2003).

14 M. P. Johansson, D. Sundholm, and J. Vaara, Angew. Chem. Int. Ed. 43, 2678 (2004).

15 M. J. X. Gu, S. H. Wei, and X. G. Gong, Phys. Rev. B 70, 205401 (2004).

16 E. Fernández, M. B. Torres, and L. C. Balbás, Progr. Theor. Chem. Phys. (Springer) 15, 407 (2006).

17 J. Wang, J. Jellinek, J. Zhao, Z. Chen, R. B. King, and P. v. R. Schleyer, J. Phys. Chem. A 109, 9265 (2005).

18 L. Xiao and L. Wang, J. Phys. Chem. A 108, 8605 (2004).

19 W. Q. Tian, M. Ge, B. R. Sahu, D. Wang, T. Yamada, and S. Mashiko, J. Phys. Chem. A 108, 3806 (2004).

${ }^{20}$ W. Q. Tian, M. Ge, B. R. Sahu, D. Wang, T. Yamada, and S. Mashiko, J. Phys. Chem. A 109, 6620 (2005).

21 P. Schwerdtfeger, Heteroatom Chemistry 13 (2002). 
22 L. Xiao and L. Wang, Chem. Phys. Lett. 392, 452 (2004).

23 R. M. Olson, S. Varganov, M. S. Gordon, H. Metiu, S. Chretien, P. Piecuch, K. Kowalski, S. Kucharski, and M. Musial, J. Am. Chem. Soc. 127, 1049 (2005).

24 V. K. Han, J. Chem. Phys. 124, 024316 (2006).

25 C. Massobrio, A. Pasquarello, and A. D. Corso, J. Chem. Phys. 109, 6626 (1998).

26 J. M. Soler, E. Artacho, J. D. Gale, A. García, P. O. J. Junquera, and D. Sánchez-Portal, J. Phys.: Condens. Matter 14, 2745 (2002).

27 B. S. de Bas, M. J. Ford, and M. B. Cortie, Journal of Molecular Structure (Theochem) 686, 193 (2004).

28 J. Wang, G. Wang, and J. Zhao, Phys. Rev. B 66, 035418 (2002).

29 A. V. Walker, J. Chem. Phys. 122, 094310 (2005).

${ }^{30}$ F. Remacle and E. S. Kryachko, J. Chem. Phys. 122, 044304 (2005).

${ }^{31}$ H. Grönbeck and P. Broqvist, Phys. Rev. B 71, 73408 (2005).

32 Z.-H. Li, D. Moran, K.-N. Fan, and P. v. R. Schleyer, J. Phys. Chem. A 109, 3711 (2005).

33 C. S. Wannere, C. Corminboeuf, Z.-X. Wang, M. D. Wodrich, R. B. King, and P. von R. Schleyer, J. Am. Chem. Soc. 127, 5701 (2005).

34 A. C. Tsipis and C. A. Tsipis, J. Am. Chem. Soc. 127, 10623 (2005).

35 A. Hirsch, Z. Chen, and H. Jiao, Angew. Chem. Int. Ed. 39, 3915 (2000).

36 W. Fa, C. Luo, and J. Dong, Phys. Rev. B 73, 085405 (2006).

37 W. Kohn and L. J. Sham, Phys. Rev. 145, 561 (1965).

38 J. P. Perdew, K. Burke, and M. Ernzerhof, Phys. Rev. Lett. 77, 3865 (1996).

39 J. P. Perdew and A. Zunger, Phys. Rev. B 23, 5075 (1981).

40 N. Troullier and J. L. Martíns, Phys. Rev. B 43, 1993 (1991).

41 L. Kleinman and D. M. Bylander, Phys. Rev. Lett. 48, 1425 (1982).

42 E. M. Fernández, M. B. Torres, and L. C. Balbás, Int. J. Quantum Chem. 99, 39 (2004).

43 O. F. Sankey and D. J. Niklewski, Phys. Rev. B 40, 3979 (1989).

44 L. C. Balbás, J. L. Martíns, and J. M. Soler, Phys. Rev. B 64, 165110 (2001).
45 A. Rubio, L. C. Balbás, and J. A. Alonso, Z. Physik D 26, 284 (1993).

46 E. Lipparini, Modern Many-Particle Physics, World Scientific, 2003, p.51.

47 M. L. Glasser and J. Boersma, SIAM J. Appl. Math. 43, 535 (1983).

48 W. Fa, C. Luo, and J. Dong, Phys. Rev. B 72, 205428 (2005).

49 Y. Gao and X. C. Zeng, J. Am. Chem. Soc. 127, 3698 (2005).

50 Formally, this number of electrons is identical to the one allowed by the spherical aromaticity rule to constitute fullerens 35 .

51 M.Torrent-Sucarrat, M. Duran, J. M. Luis, and M. Solà, J. Phys. Chem. A 109, 615 (2005).

52 B. O. Roos, R. Lindh, P.-A. Malmqvist, V. Veryazov, and P.-O. Widmark, J. Phys. Chem. 109, 6575 (2005).

${ }^{53}$ P. Neogrady, V. Kello, M. Urban, and A. J. Sadlej, Int. J. Quantum Chem. 63, 557 (1997).

54 A. Castro, Ph.D. thesis, Universidad de Valladolid, Spain (2004).

55 T. Saue and H. J. A. Jensen, J. Chem. Phys. 118, 522 (2003).

56 A. Castro, M. A. L. Marques, J. A. Alonso, and A. Rubio, J. Comput. Nanosci. 1, 231 (2004).

57 J. Zhao, J. Yang, and J. G. Hou, Phys. Rev. B 67, 085404 (2003).

58 E. Benichou, R. Antoine, D. Rayane, B. Vezin, F. W. Dalby, P. Dugourd, M. Broyer, C. Ristori, F. Chandezon, B. A. Huber, et al., Phys. Rev. A 59, R1 (1999).

59 D. Rayane, A. R. Allouche, E. Benichou, R. Antoine, M. Aubert-Frecon, P. Dugourd, M. Broyer, C. Ristori, F. Chandezon, B. A.Huber, et al., Eur. Phys. J. D 9, 243 (1999).

60 K. R. S. Chandrakumar, T. K. Ghanty, and S. K. Ghosh, J. Phys. Chem. A 108, 6661 (2004).

61 M. Yang and K. A. Jackson, J. Chem. Phys. 122, 184317 (2005).

62 A. Mañanes, M. Membrado, A. F. Pacheco, J. Sañudo, and L. C. Balbás, Int. J. Quantum Chem. 52, 767 (1994).

63 J. P. Perdew, Phys. Rev. B 37, 6175 (1988).

64 I. Vasiliev, S. Öğüt, and J. R. Chelikowsky, Phys. Rev. Lett. 78, 4805 (1997). 